\title{
Image Force Effect on the Separation of Partial Dislocations in Bicrystals of Hexagonal Structure $\mathrm{Zn}-\mathrm{Tl}$ and $\mathrm{Zn}-\mathrm{Be}$
}

\author{
A. Ayadi and O. Khalfallah \\ Laboratory of Microstructures and Defects in Materials, Mentouri University, Constantine, Algeria
}

\begin{abstract}
We study the elastic interaction between a pair of partial dislocations, resulting from the dissociation of a perfect dislocation, and a bimetallic interface. The forces that act on two partials dislocations are the forces due to elastic interaction between the partial and image forces due to interactions of partial dislocations with interface. We are interested in the effect of image force on width of the stacking fault ribbon between two Schockley partials. We show that the separation of two partials dislocations is modified compared to that in the single crystal. It depends on the ratio of shear modulus and the distance between the interface and the dislocation.
\end{abstract}

DOI: 10.12693/APhysPolA.123.302

PACS: 61.72.Lk, 61.72.Mm, 61.72.Nn, 62.20.D-

\section{Introduction}

The perfect dislocations dissociate into partial dislocations with creating a stacking fault. The differences in mechanical behavior between the materials come from the ease or difficulty for dislocations to dissociate. When the dislocations are dissociated, they lose their mobility and the material presents high work hardening ability. We can thus characterize metals and alloys due to their stacking fault energy which is measured from the distance of separation of partial dislocations. This energy was derived by Read [1] for isotropic medium and by Chou and Eshelby [2] for an anisotropic crystal, and it depends on the crystal structure. This energy was derived by Read [1] for isotropic medium and by Chou and Eshelby [2] for an anisotropic crystal, and it depends on the crystal structure. This energy is denoted $\gamma_{s}$ and is inversely proportional to the distance between the partials. Studies of elastic interactions between dislocations and interface made by Priester and Khalfallah [3-5] showed that the image force in elastically anisotropic materials plays an important role in the dislocation movement vis-à-vis the interface. As well analysis of the image force exerted on a dislocation near a bimetallic interface was take in detail by Dundurs and Sendeckyj [6], Lin and Lee [7] and Lin [8]. For this purpose we investigate the behavior of dissociated dislocations near the interface.

\section{Configuration of dislocations and studied materials}

An edge perfect dislocation, the Burgers vector $\boldsymbol{b}$, located in the basal plane (0001) of a material with hexagonal structure, can dissociate into a pair of Schockley partial dislocations with the Burgers vectors $\boldsymbol{b}_{1}$ and $\boldsymbol{b}_{2}$ according to the following:

$$
\frac{a}{3}[11 \overline{2} 0] \rightarrow \frac{a}{3}[10 \overline{1} 0]+\frac{a}{3}[01 \overline{1} 0] .
$$

The leading partial dislocation is located at distance $d$ of the interface and the queue partial dislocation is at distance $(d+l)$ from the interface, Fig. 1. They are parallel to the interface and are in elastic interaction with it. The interface is defined by its plane which is the basal plane for the two crystals and its disorientation which is null.

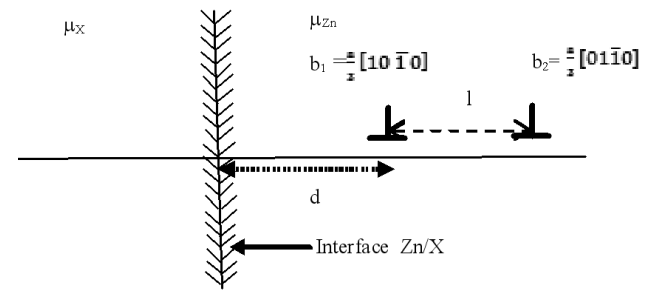

Fig. 1. Schematic of two partial dislocations near the interface $\mathrm{Zn}-\mathrm{X} . l$ - width of separation between two Schockley partials, $d$ - distance between the interface and the leading partial dislocation.

The bicrystal consists of two metals of hexagonal structure, the first is zinc and the second is beryllium or tellurium. The two metals are selected to obtain ratios of shear modulus $\mu_{2} / \mu_{1}>1$ for Zn-Be bicrystal and $\mu_{2} / \mu_{1}<1$ for $\mathrm{Zn}-\mathrm{Tl}$ where $\mu_{\mathrm{Zn}}=46.6 \mathrm{GPa}$, $\mu_{\mathrm{Be}}=149.9 \mathrm{GPa}$ and $\mu_{\mathrm{Tl}}=6.2 \mathrm{GPa}[9,10]$. The two partial dislocations are located in zinc crystal.

\section{Results and discussion}

Determining the separation distance between the two partials, in interaction with the interface, requires the calculation of forces acting on them: the elastic interaction force, $F_{\mathrm{el}}$, by the Peach-Koehler law and the image force, $F_{\mathrm{i}}$, by the theorem of Barnett and Lothe [9].

- The force due to elastic interaction, $F_{\mathrm{el}}$, between the partials is calculated in a single crystal of zinc. It controls the separation distance, $l$, of the two partials:

$$
F \mathrm{e} l=\mu_{\mathrm{Zn}} b^{2} / 2 \pi l .
$$

- The image forces, $F_{\mathrm{i} 1}$ and $F_{\mathrm{i} 2}$, exerted on each partial. They are controlled by their distances from the interface: $d$ for the leading partial and $(d+l)$ for the queue 
partial

$$
\begin{aligned}
& F_{\mathrm{i} 1}=-\frac{E_{\mathrm{I} / \mathrm{II}}-E_{\mathrm{I}}}{d}=-\frac{\Delta E}{d}, \\
& F_{\mathrm{i} 2}=-\frac{E_{\mathrm{I} / \mathrm{II}}-E_{\mathrm{I}}}{d+l}=-\frac{\Delta E}{d+l},
\end{aligned}
$$

where $\Delta E$ is the elastic energy of dislocation-interface interaction. $F_{\mathrm{i} 1}(\mathrm{Eq} .(3))$ is the image force corresponding to the leading partial dislocation is located at distance $d, F_{\mathrm{i} 2}$ (Eq. (4)) is the image force corresponding to the queue partial dislocation is at distance $(d+l)$ from the interface.

The difference between the intensities of images forces that are exerted on each of the two partial $\Delta F_{\mathrm{i}}$, is due to the different distances that separate the two partial dislocations of the interface, $d$ and $(d+l)$. It induces the alignment or combination between the two partial dislocations according to the image force is attractive or repulsive. ( the effect of the image force, it decreases or increases the separation of the two partial? !) so it depends on the sign and intensity of the difference of the image force which is corresponding $\Delta E$.

The new partial separation $l^{\prime}$ under the effect of the image force is obtained by the relation:

$$
F_{\mathrm{el}}=\left(\mu_{\mathrm{Zn}} b^{2} / 2 \pi l\right)+\Delta F_{\mathrm{i}}=\mu_{\mathrm{Zn}} b^{2} /\left(2 \pi l^{\prime}\right) .
$$

We take the force of interaction misfit dislocationpartial dislocation as a constant.

The elastic interaction energies, $\Delta E$, of the Shockley partial dislocations with interface for the bicrystals $\mathrm{Zn}-\mathrm{Be}$ and $\mathrm{Zn}-\mathrm{Tl}$ are shown in Table.

\section{TABLE}

Ratios of shear modulus and elastic interaction energies between partial dislocations and interface, $\Delta E$, for the bicrystals $\mathrm{Zn}-\mathrm{Be}$ and $\mathrm{Tl}-\mathrm{Zn}$.

\begin{tabular}{c|c|c}
\hline \hline Bicrystal & Zn-Be & Zn-Tl \\
\hline$\mu_{2} / \mu_{1}$ & 3.206 & 0.133 \\
$\Delta E[\mathrm{pJ} / \mathrm{m}]$ & 58.153 & -164.326
\end{tabular}

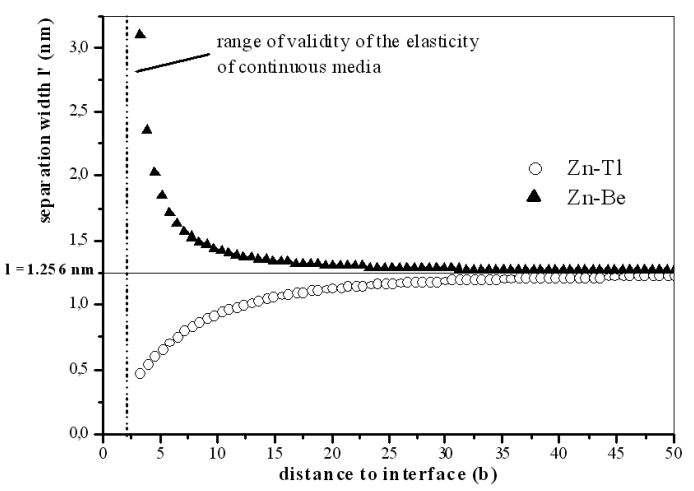

Fig. 2. Effect of image force on separation of partials. $l=1.256 \mathrm{~nm}$ is the equilibrium separation of partials in a single crystal of $\mathrm{Zn}$.

When the interaction concerns a dissociated dislocation, the width of separation between the two partial dislocations is obtained by Eq. (5) and represented in Fig. 2 according to distance $d$ from the interface.

In $\mathrm{Zn}-\mathrm{Tl}$ bicrystal $\left(\mu_{2} / \mu_{1}<1\right)$, where $\mathrm{Tl}$ is softer than zinc, the interaction energy is negative and the image force is attractive. Dislocations located at a distance less than the efficacy distance of image force are attracted to the interface. Figure 2 shows that width of separation between the Schockley partials decreases as they approach the interface. The image force favors partial recombination particularly when the partials are closer to the interface.

In $\mathrm{Zn}-\mathrm{Be}$ bicrystal $\left(\mu_{2} / \mu_{1}>1\right)$, where $\mathrm{Be}$ is harder than zinc, the elastic interaction energy is positive and the image force is repulsive. Dislocations at distances of interface less than the efficacy distance of image force is repelled. The separation of partial increases as they approach the interface, it passes from $1.2 \mathrm{~nm}$ to $3 \mathrm{~nm}$ for the distance corresponding to the limit of validity of linear elasticity of continuous media. The image force favors the separation of partials and prevents their recombination. These results agree with those of Lin [8].

\section{Conclusion}

The effect of image force plays an important role on interaction of partial dislocations with bimetallic interface in case of bicrystal of hexagonal structure. The image force modifies the equilibrium separation of partials compared to that in a single crystal. Separation of the two partial dislocations depends on the ratio of shear modulus of the two materials which constitute the bicrystal. The stacking fault ribbon issued from the dissociation of a perfect dislocation is expanded under effect of image force for a dislocation close to second harder crystal. The image effect decreases stacking fault energy of first crystal which contains the dislocation. The same ribbon is narrowed when the second crystal is softer than the first, then the stacking fault energy is increased under the influence of the image force.

\section{References}

[1] W.T. Read, Dislocations in Crystals, McGraw-Hill, New York 1953, p. 131.

[2] Y.T. Chou, J.D. Eshelby, J. Mech. Phys. Solids 10, 27 (1962)

[3] L. Priester, Mater. Sci. Eng. A 309-310, 430 (2001).

[4] L. Priester, O. Khalfallah, Philos. Mag. A 69, 471 (1994)

[5] L. Priester, O. Khalfallah, A. Coujou, Philos. Mag. A 75, 61 (1997)

[6] J. Dundurs, G.P. Sendeckyj, J. Appl. Phys. 36, 3353 (1965)

[7] K.M. Lin, S. Lee, Phys. Status Solidi A 120, 497 (1990)

[8] K.M. Lin, Mater. Sci. Eng. A 165, 109 (1993)

[9] D.M. Barnett, J. Lothe, J. Phys. F 4, 1618 (1974)

[10] H.B. Huntington, The Elastic Constants of Crystals, Academic Press, New York 1958, p. 66. 